\title{
The predictive ability of plasma ESRI mutations for the efficacy of endocrine therapy in hormone- receptor-positive advanced breast cancer
}

This article was published in the following Dove Press journal: OncoTargets and Therapy

\author{
Yangfan Du' \\ $\mathrm{Na} \mathrm{Li}$ \\ Xin Jiao ${ }^{2}$ \\ Kai Li' \\ Shunchao Yan' \\ 'Department of Oncology, Shengjing \\ Hospital of China Medical University, \\ Shenyang I I 0022, People's Republic \\ of China; ${ }^{2}$ Department of Respiratory \\ Medicine, Shenyang Chest Hospital, \\ Shenyang II 0044, People's Republic \\ of China
}

Purpose: The predictive ability of plasma ESR 1 mutations for outcomes among patients with advanced breast cancer undergoing endocrine therapy (ET) remains disputable. We performed a comprehensive meta-analysis of published studies to clarify the impact of plasma ESR1 mutations on clinical outcomes for patients after subsequent ET.

Materials and methods: An electronic search was performed to identify eligible studies. Studies analyzing progression-free survival (PFS) and/or overall survival (OS) according to plasma ESR1 mutation status after subsequent ET were included. HRs were calculated using a fixed- or random-effects model according to heterogeneity. Pooled HRs and 95\% CIs were used to estimate the effects.

Results: Six studies including 705 patients with advanced breast cancer met the inclusion criteria. The impact of plasma ESR1 mutations on PFS and OS after subsequent ET was reported in six studies (seven groups) and two studies, respectively. Meta-analysis results showed that the pooled HR for ESR1 mutations was 1.70 (95\% CI, 1.05-2.74; $P=0.03)$ for OS, which was statistically significant for predicting poor survival, and 1.56 (95\% CI, 1.13-2.14; $P=0.006)$ for PFS; however, Begg's and Egger's test results identified the presence of bias. The trim-and-fill method was used, and after incorporation of the imputed studies, the HR was 1.16 (95\% CI, 0.88-1.53, P=0.30) for PFS, which indicates that plasma ESR1 mutation had no effect on PFS after subsequent ET. Subgroup analysis suggested that plasma ESRI mutations were correlated with shorter PFS (HR, $1.98 ; 95 \% \mathrm{CI}, 1.12-3.51 ; P=0.02$ ) in patients subsequently treated with aromatase inhibitors (AIs), whereas no association with PFS was observed for patients subsequently treated with non-AI ET (HR, 1.08; 95\% CI, 0.85-1.38; $P=0.54$ ) or fulvestrant (HR, 1.03; 95\% CI, 0.79-1.34; $P=0.83$ ).

Conclusion: The current meta-analysis demonstrates that plasma ESR1 mutation status is not a predictor of ET efficacy for all drugs without distinction in patients with hormone-receptorpositive advanced breast cancer. ESRI mutation predicted a poor response to AIs, whereas it was not predictive of non-AI ET efficacy, especially for fulvestrant.

Keywords: ESR1 mutation, breast cancer, endocrine therapy, efficacy predictor

\section{Introduction}

Breast cancer $(\mathrm{BC})$ is a heterogeneous disease that is classified into five molecular subtypes according to estrogen receptor (ER), progesterone receptor (PR), HER-2, and Ki-67 status. ${ }^{1}$ Approximately $70 \%$ of BCs are ER positive, and survival depends on estrogen signaling pathways. ${ }^{2}$ Endocrine therapy (ET) targeting ER activity is an effective strategy for hormone-receptor-positive $\mathrm{BC}$, especially luminal A-type cancers. ET is the first choice for the treatment of patients with ER-positive metastatic BC (MBC) who only have bone metastases or asymptomatic visceral metastasis. ${ }^{3}$ However, ER-positive
Correspondence: Shunchao Yan Department of Oncology, Shengjing Hospital of China Medical University, No. 39 Huaxiang Road, Tiexi District, Shenyang I 10022, People's Republic of China

Tel/fax +8624966 I56 3II 5

Email yanshunchao666@I63.com 
MBC patients who are treated with ET often display markedly different responses. Relapse occurs within months after treatment in certain patients, whereas in others, relapse occurs after many years. Therefore, the identification of new markers to distinguish patients according to the response to treatment would help in improving the design of effective therapies. The ER is encoded by the ESR I gene, and ESR I mutations were first studied in BC cells in 1997. ${ }^{4}$ Ligand-binding domain mutations, which are the most frequent ESR1 mutations, cause ligandindependent activation of the ER and confer ET resistance. ${ }^{5,6}$ Several recent studies showed that ESR 1 mutations occur preferentially in patients previously treated with ET, particularly aromatase inhibitors (AIs). ${ }^{7}$ Biopsied metastatic tissues were initially used to determine the prevalence of ESR 1 mutations using next-generation sequencing (NGS). ${ }^{8-11}$ Because of the limitations (invasive and heterogeneous) of metastatic biopsies, ESR 1 mutations are assessed using liquid biopsies based on the new consensus that circulating tumor DNA (ctDNA) is a noninvasive substitute for metastasis biopsy. ${ }^{12-17}$ Recently, studies have suggested that the presence of ESR1 mutations is related to poor subsequent ET efficacy. ${ }^{16,17}$ However, other studies showed that ESR1 mutations are not associated with the efficacy of subsequent ET. ${ }^{12-15}$ Although the role of ESR1 mutations in predicting the efficacy of ET has been discussed extensively, no consensus has been reached to date. A systematic study of published data is necessary to provide high-level evidence-based guidance. In the current study, we collected eligible data on the role of ESR1 mutations in predicting ET efficacy to clarify the value of ESR 1 status detection for clinical decision-making regarding subsequent ET.

\section{Materials and methods}

\section{Search strategy and selection criteria}

The medical electronic databases such as PubMed, Web of Science, Embase, and the Cochrane Register of Controlled Trials (CENTRAL) were searched using the keywords "ESR1 mutation", "breast cancer", "endocrine therapy", "selective estrogen receptor down-regulators (SERDs)", "fulvestrant", "selective estrogen receptor modifiers (SERMs)", "tamoxifen", and "aromatase inhibitor" to identify studies investigating the role of ESR 1 mutation status in the efficacy of BC ET (last search updated on March 28, 2018). ctDNA analysis in plasma was used to identify ESR 1 mutations. If the study involved several arms, each valid arm was considered separately. Irrelevant studies were first excluded by reading the titles and abstracts. Then, the remaining articles were carefully read to identify eligible studies. The references of all retrieved articles were manually searched to identify other potentially relevant studies.

\section{Data extraction}

Two authors (YD and NL) performed the electronic and manual searches independently, and the inclusion of an article was decided by consensus. The two authors extracted the following information from each report independently: name of the first author, publication year, patient cohort size, source of patients, previous ET, the rate of ESR1 mutations, subsequent treatment, assessment methods for ESR 1 mutations, and HRs for progression-free survival (PFS) or overall survival (OS). The two authors then analyzed the studies together to identify potential differences in data extraction. Guidelines of the Cochrane reviewers' handbook were used to assess the quality of the studies. ${ }^{18}$

\section{Statistical analyses}

HRs with $95 \%$ CIs were used for pooled data. For studies that did not directly provide HRs and 95\% CIs, Kaplan-Meier survival curves and related data were used to calculate the HRs and 95\% CIs using the methods described by Parmar et $\mathrm{al}^{19}$ and Tierney et al. ${ }^{20}$ Data were extracted from KaplanMeier curves using Engauge Digitizer version 2.11 (free software can be downloaded from http://sourceforge.net). An HR value of $>1$ reflects a worse outcome. The heterogeneity of the studies was assessed using Cochran's test. A $P$-value of $<0.05$ was considered to indicate statistically significant heterogeneity, and the random-effects model was applied. The fixed-effects model was applied if there was no significant heterogeneity. The statistical tests were performed using RevMan version 5.3 (free software can be downloaded from http://www.cochrane.org). Begg's and Egger's tests were performed using Stata SE 12.0 (StataCorp LP, College Station, TX, USA).

\section{Results Description of studies}

As shown in Figure 1, 353 records were selected using our search strategy. After reading the titles and abstracts, 329 studies were excluded because they were review articles, basic studies, case reports, or irrelevant to our analysis. Ten additional articles were excluded, because data were unavailable for validation or because of repeated data. Finally, six eligible studies published between 2015 and 2017 were included in the current analysis. ${ }^{12-17}$ All the studies included determined ESR 1 mutations in ctDNA from plasma samples using the droplet digital polymerase chain reaction (ddPCR) method. Baseline blood samples were drawn from patients before ET initiation. Fribbens et $\mathrm{al}^{14}$ analyzed the role of ESR1 mutations in two groups according to ET agents: one group was treated with exemestane and the other with fulvestrant. 


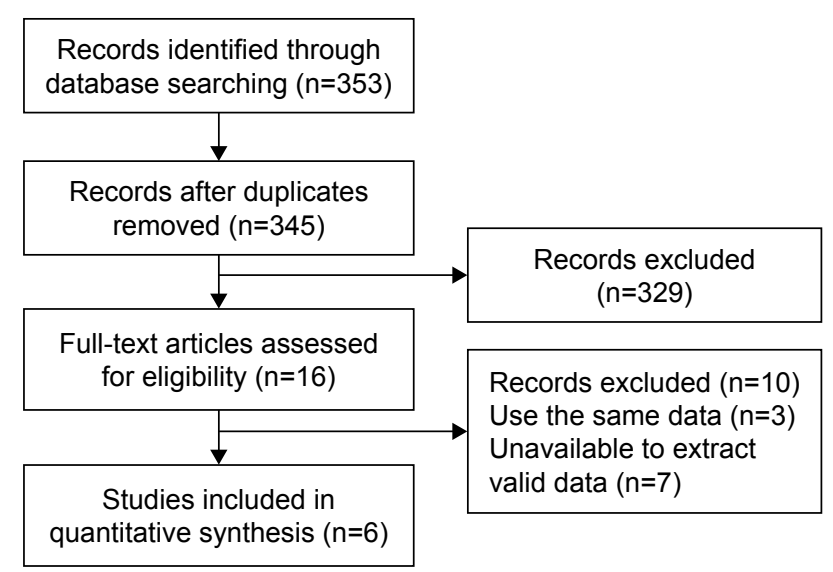

Figure I Brief flowchart.

For this study, we extracted separate HRs for the two groups. The characteristics of the six studies (seven groups) are summarized in Table 1. The prevalence of ESR1 mutations ranged from $15.6 \%$ to $43 \%$. The number of participants varied from 45 to 224. In total, 705 patients were included in our analysis. Clatot et al. ${ }^{12}$ reported that one patient was refractory to $\mathrm{CT}$ scan and refused some of the exams in their study. Thus, they could not determine the PFS and only interpreted the OS. This patient had ESR1 mutation and was treated with tamoxifen. So, when describing PFS, data were reported for 50 patients, and one patient was not evaluable. When describing OS, data were reported for 51 patients. All the patients included had MBC, and most of them had a history of prior ET.

\section{Impact of ESRI mutations on ET outcomes in BC}

PFS was assessed in seven groups comprising a total of 704 patients. As shown in Figure 2, there was significant heterogeneity among the studies $\left(P=0.004, P^{2}=69 \%\right)$, and a random-effects model was applied. The pooled HR was 1.56 (95\% CI, 1.13-2.14; $P=0.006$ ), indicating that $E S R 1$ mutation was associated with shorter PFS. Two studies involving 108 cases investigated OS. The heterogeneity was not significant $(P=0.92, P=0 \%)$. A fixed-effects model was applied to combine HRs. The pooled HR was 1.70 (95\% CI, 1.05-2.74; $P=0.03$ ), indicating that $E S R 1$ mutation was associated with shorter OS.

\section{Impact of ESRI mutation on outcomes for Al-treated and non-Al-treated BC}

Subgroup analysis was performed according to subsequent treatment with ET agents. Three studies (291 cases) addressing patients subsequently treated with AIs were analyzed. As shown in Figure 3A, a random-effects model was adopted because there was significance between-study heterogeneity ( $\left.P=0.01, I^{2}=78 \%\right)$. The pooled HR was 1.98 (95\% CI, 1.12-3.51; $P=0.02)$, indicating that ESR1 mutation was associated with worse PFS in patients receiving subsequent AI treatment. Three studies (344 cases) described patients subsequently receiving non-AI (fulvestrant or tamoxifen) treatment. The fixed-effects model was applied because there was no heterogeneity $\left(P=0.54, I^{2}=0 \%\right)$ among these studies. As shown in Figure 3B, the combined HR for PFS was 1.08 (95\% CI, 0.85-1.38; $P=0.54$ ). PFS was assessed for 294 $\mathrm{MBC}$ cases in two studies according to subsequent fulvestrant treatment. There was no heterogeneity between the studies ( $P=0.55, I^{2}=0 \%$ ), and a fixed-effects model was used. The combined HR for PFS was 1.03 ( $95 \% \mathrm{CI}, 0.79-1.34 ; P=0.83$ ). The results indicate that ESR1 mutations did not influence PFS among patients treated with fulvestrant or non-AI ET.

\section{Publication bias}

The publication bias was assessed using Begg's and Egger's tests. PFS was evaluated in seven groups of MBC patients treated with ET. A funnel plot exhibited visual inspection asymmetry. Results for Begg's test $(P=0.016)$ and Egger's test $(P=0.004)$ showed statistically significant bias (Figure 4A). The trim-and-fill method was applied, and three hypothetical studies were imputed. Funnel plot symmetry was analyzed for ten groups (Figure 4B). The analysis incorporating the imputed studies yielded different data from the original result. The combined HR was 1.16 (95\% CI, 0.88-1.53; $P=0.30$ ). The result demonstrated that $E S R 1$ mutation was not associated with PFS according to the studies analyzed. No publication bias was detected in other subgroups.

\section{Discussion}

Hormone-receptor-positive advanced $\mathrm{BC}$ is the most frequent $\mathrm{BC}$ subtype. Estrogen is the most important hormone stimulating this type of $\mathrm{BC}$ cell. The ER is activated and promotes $\mathrm{BC}$ cell proliferation after being bound by estrogen. ET affects different stages of the estrogen signaling pathway, and its efficacy in the treatment of $\mathrm{BC}$ has been extensively demonstrated. ET has fewer serious side effects and a higher therapeutic efficacy than other systemic treatments in hormone-receptor-positive MBC. It is the preferred treatment for hormone-receptor-positive MBC without life-threatening visceral metastases. However, after ET treatment, BC cells often acquire new survival signals or undergo genomic changes conferring resistance to ET. The era of precision medicine has changed the treatment of several cancers. However, there are no effective biomarkers other than ER, PR, and HER-2 for predicting the efficacy of ET. ${ }^{21}$ ESR 1 , the gene encoding the 
ER, undergoes mutations in response to ET. Several recent studies indicate that ESR1 mutation status may be a predictive biomarker for the response to ET. Many studies detected ESR1 mutations using NGS before 2015. ${ }^{8-11}$ Most recently, multiplex digital PCR assays have been used to assess ESR 1 mutation status in liquid biopsies. ${ }^{12-17}$ In the current study, we analyzed six studies ( 705 cases) published between 2015 and 2017, all of which assessed ESR1 mutation status using ddPCR. The results of our analysis show that the risk of death was significantly higher (by 70\%) after subsequent ET among patients with ESR 1 mutations compared to patients with wildtype ESR 1; however, these results were based on 108 cases. Although a $56 \%$ increase in recurrence was observed among ESR1 mutation patients after subsequent ET, the Begg's and Egger's test results identified a publication bias. Imputation of three hypothetical studies using the trim-and-fill method changed the PFS results, indicating that the presence of ESR1 mutation did not influence PFS after subsequent ET. These results indicate that ESR1 mutations may result in a worse outcome after subsequent ET; however, the number of cases was too small, and further larger studies are needed to verify these results. In addition, the subsequent ET drugs differed among the six studies. Tamoxifen, AIs, and fulvestrant act at different stages of estrogen signaling. This may introduce bias into the analysis. A subgroup meta-analysis was therefore performed to avoid the impact of this bias.

AIs have replaced tamoxifen in the postoperative adjuvant therapy of postmenopausal BC since the 1990s. ${ }^{21}$ The SOFT and TEXT studies and our further meta-analysis showed that the suppression of ovarian function in combination with AIs was an efficacious adjuvant therapy for ER-positive premenopausal $\mathrm{BC}$ with a high risk of recurrence. ${ }^{22,23}$ In ER-positive MBC, AIs are also the first ET choice in patients without prior exposure to AIs. Studies have shown that ESR 1 mutation rarely occurs in primary $\mathrm{BC}$, whereas it frequently appears in patients previously treated with AIs. ${ }^{13,16}$ AIs act by depriving BC cells of estrogen. The ligand-binding domain is the most frequent ESR1 mutation site. These mutations result in constitutive ER activity even in the absence of estrogen and theoretically reduce the efficacy of AIs. The current meta-analysis results show that ESR1 mutations led to a $98 \%$ increase in the risk of recurrence among patients subsequently treated with AIs. These results identify the ESRI mutation as a biomarker of AI resistance and predict poor efficacy of subsequent AI treatment. Decisions regarding subsequent lines of AI treatment should at least in part be based on the detection of circulating ESR1 mutations in patients with MBC who 
A

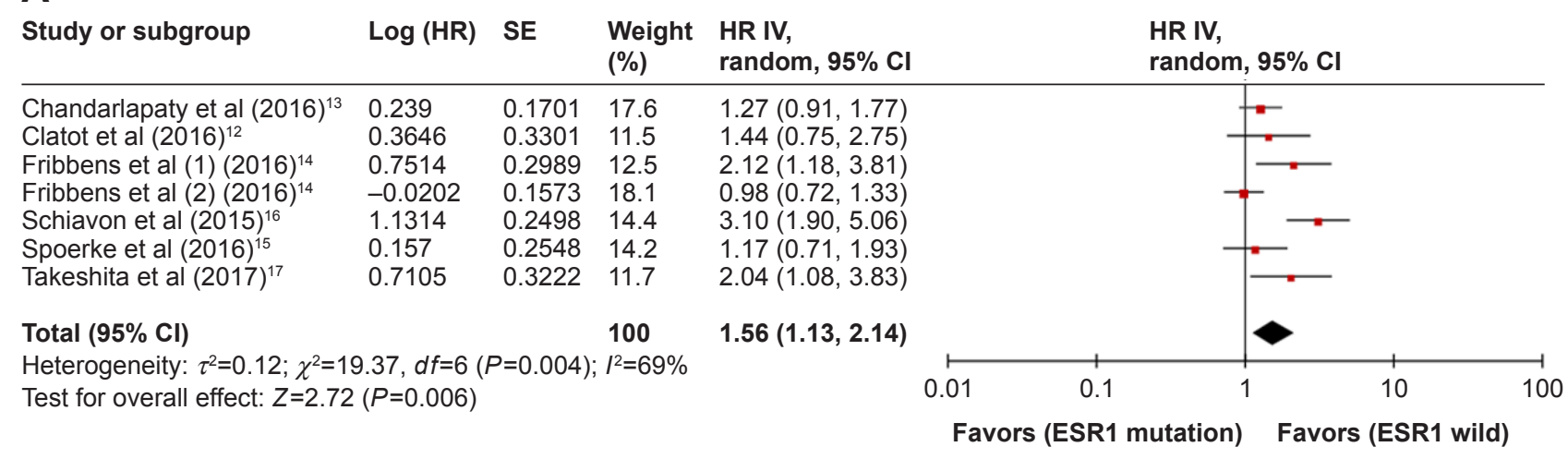

B

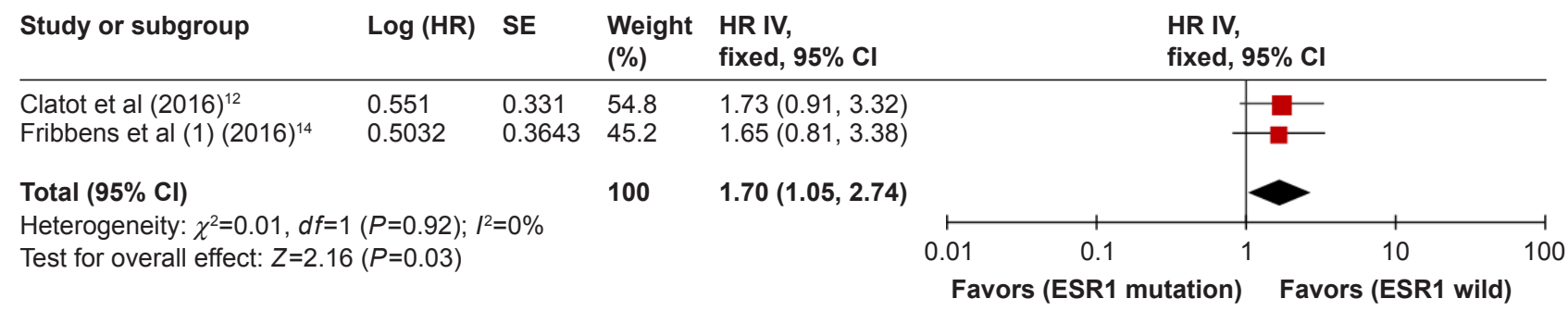

Figure 2 Forest plot of HR for PFS (A) and OS (B) of all included BC patients.

Abbreviations: BC, breast cancer; IV, inverse variance; OS, overall survival; PFS, progression-free survival; SE, standard error.

A

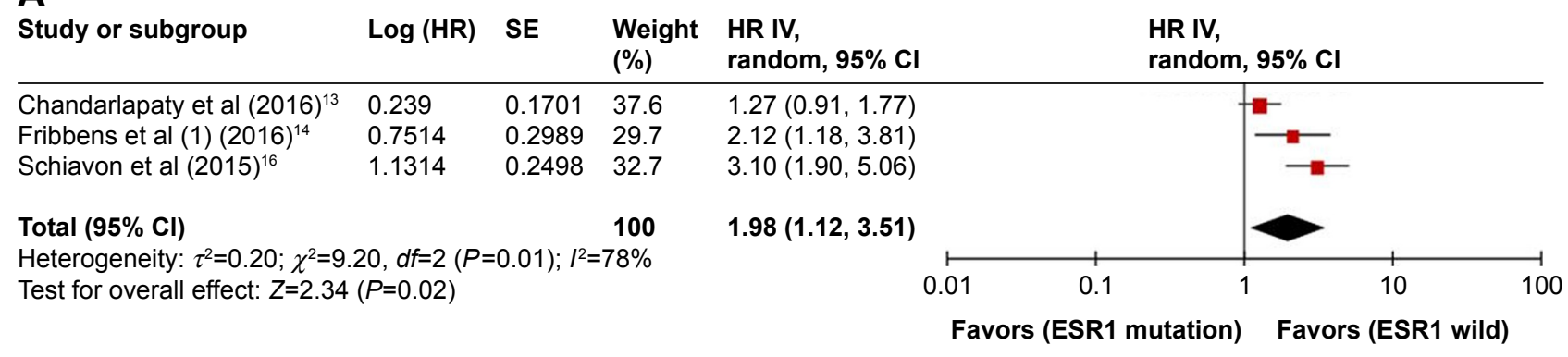

B

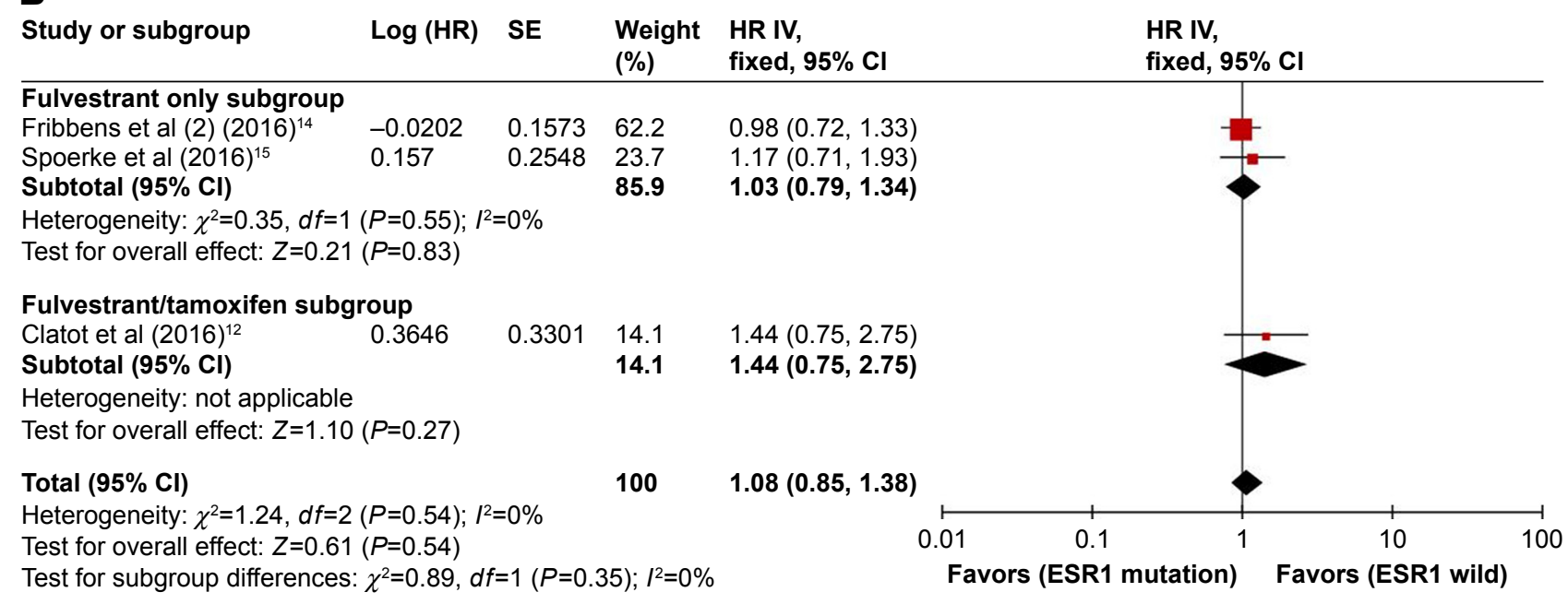

Figure 3 Forest plot of HR for PFS of subsequently Al-treated (A) and non-Al-treated (B) patients.

Abbreviations: Al, aromatase inhibitor; IV, inverse variance; PFS, progression-free survival; SE, standard error. 
A

Begg's funnel plot with pseudo $95 \%$ confidence limits

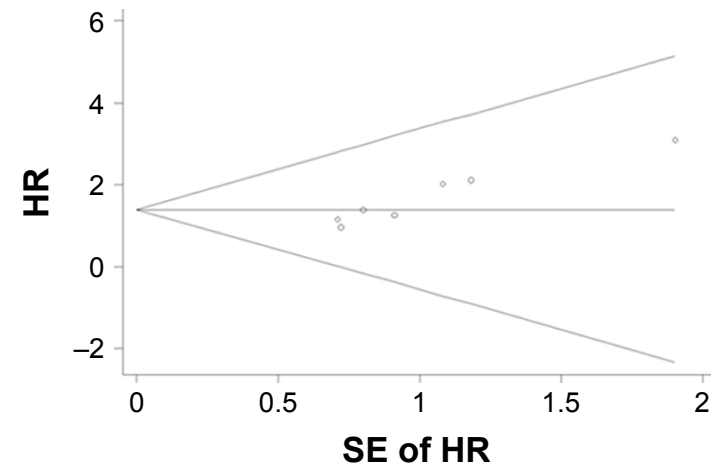

B Filled funnel plot with pseudo $95 \%$ confidence limits

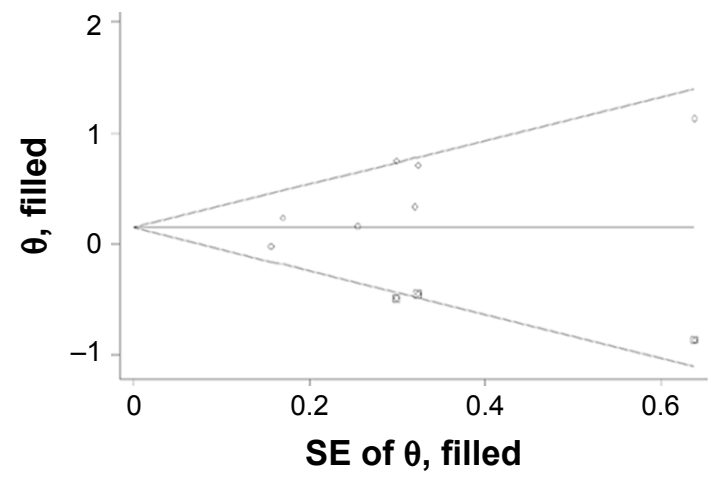

Figure 4 Funnel plot without (A) and with (B) trim and fill for PFS of all included BC patients. Abbreviations: BC, breast cancer; PFS, progression-free survival; SE, standard error.

show disease progression after treatment with antiestrogens or estrogen deprivation therapies.

Fulvestrant, a selective ER degrader, is associated with the longest PFS when used as first-line single-agent ET in HRpositive MBC. ${ }^{24}$ Fribbens et $\mathrm{al}^{14}$ analyzed the ESR 1 mutation status of patients from the SoFEA and PALOMA-3 studies. We extracted PFS data for patients who received fulvestrantcontaining regimens from the SoFEA trial and patients who received fulvestrant plus placebo in the PALOMA-3 trial. To avoid bias, the fulvestrant + palbociclib arms were not included. The meta-analysis results indicate that ESR1 mutation status had no effect on outcomes after fulvestrant treatment. This demonstrates that ESR 1 mutation status is not associated with fulvestrant sensitivity. Clatot et $\mathrm{al}^{12}$ evaluated the effect of ESR1 mutation on tamoxifen or fulvestrant efficacy. We performed a subgroup meta-analysis for the non-AI subsequent treatment group. The results reveal that ESR1 mutation status had no influence on the efficacy of non-AI ET. These findings indicate that ESR 1 mutation has no predictive value among patients treated with non-AI ET.

Our current analysis included the latest studies, and we performed further subgroup analyses to provide more valid and personalized results. However, our study also has some limitations. It is regrettable that only two to three studies were included in the subgroup analyses because liquid biopsy detection of ESR 1 mutations only became popular after 2015, and little research has been performed. The study by Fribbens et $\mathrm{al}^{14}$ provided more cases than the other studies and had a relatively greater impact on the statistical results, which may reduce the authority of the current study.

\section{Conclusion}

The current meta-analysis demonstrates that ESRI mutation status is not a predictor of the efficacy of all ET drugs without distinction according to the published data. Regarding AIs, ESR 1 mutation predicted a poor response, while ESR1 mutation status had no effect on non-AI ET outcomes, especially for patients treated with fulvestrant. However, any application of these results to clinical practice requires caution because they are based on a relatively small sample size. We propose that ESR1 mutation status should be assessed in patients with MBC subsequently treated with AIs. The detection of ESR1 mutations in plasma could be used as a noninvasive method for measuring AI resistance in $\mathrm{MBC}$. Patients positive for ESR 1 mutations should not receive AIs as subsequent treatment. Large randomized controlled trials are required to verify these results.

\section{Acknowledgment}

This study was supported by grants from Science and Technology Foundation of Liaoning Province (20170540995), Science and Technology Foundation of Shenyang City (RC170545), and the National Science Foundation of China (81302313).

\section{Disclosure}

The authors report no conflicts of interest in this work.

\section{References}

1. Yersal O, Barutca S. Biological subtypes of breast cancer: prognostic and therapeutic implications. World J Clin Oncol. 2014;5(3):412-424.

2. Clark GM, Osborne CK, Mcguire WL. Correlations between estrogen receptor, progesterone receptor, and patient characteristics in human breast cancer. J Clin Oncol. 1984;2(10):1102-1109.

3. Watanabe J, Hayashi T, Tadokoro Y, Nishimura S, Takahashi K. Clinical pattern of primary systemic therapy and outcomes of estrogen receptorpositive, HER2-negative metastatic breast cancer: a review of a single institution. Breast Cancer Res Treat. 2017;166(3):911-917.

4. Zhang QX, Borg A, Wolf DM, Oesterreich S, Fuqua SA. An estrogen receptor mutant with strong hormone-independent activity from a metastatic breast cancer. Cancer Res. 1997;57(7):1244-1249. 
5. Jeselsohn R, Buchwalter G, De Angelis C, et al. ESR1 mutations - a mechanism for acquired endocrine resistance in breast cancer. Nat Rev Clin Oncol. 2015;12(10):573-583.

6. Reinert T, Gonçalves R, Bines J. Implications of ESR1 Mutations in Hormone Receptor-Positive Breast Cancer. Curr Treat Options Oncol. 2018;19(5):24.

7. Garcia-Murillas I, Schiavon G, Weigelt B, et al. Mutation tracking in circulating tumor DNA predicts relapse in early breast cancer. $\mathrm{Sci}$ Transl Med. 2015;7302(302):302ra133.

8. Merenbakh-Lamin K, Ben-Baruch N, Yeheskel A, et al. D538G mutation in estrogen receptor- $\alpha$ : A novel mechanism for acquired endocrine resistance in breast cancer. Cancer Res. 2013;73(23):6856-6864.

9. Robinson DR, Wu YM, Vats P, et al. Activating ESR1 mutations in hormone-resistant metastatic breast cancer. Nat Genet. 2013;45(12): 1446-1451.

10. Toy W, Shen $\mathrm{Y}$, Won H, et al. ESR1 ligand-binding domain mutations in hormone-resistant breast cancer. Nat Genet. 2013;45(12):1439-1445.

11. Jeselsohn R, Yelensky R, Buchwalter G, et al. Emergence of constitutively active estrogen receptor- $\alpha$ mutations in pretreated advanced estrogen receptor-positive breast cancer. Clin Cancer Res. 2014;20(7): 1757-1767.

12. Clatot F, Perdrix A, Augusto L, et al. Kinetics, prognostic and predictive values of ESR1 circulating mutations in metastatic breast cancer patients progressing on aromatase inhibitor. Oncotarget. 2016;7(46): 74448-74459

13. Chandarlapaty S, Chen D, He W, et al. Prevalence of ESR 1 mutations in cell-free DNA and outcomes in metastatic breast cancer: a secondary analysis of the BOLERO-2 clinical trial. JAMA Oncol. 2016;2(10): $1310-1315$.

14. Fribbens C, O'Leary B, Kilburn L, et al. Plasma ESR1 mutations and the treatment of estrogen receptor-positive advanced breast cancer. J Clin Oncol. 2016;34(25):2961-2968.
15. Spoerke JM, Gendreau S, Walter K, et al. Heterogeneity and clinical significance of ESR1 mutations in ER-positive metastatic breast cancer patients receiving fulvestrant. Nat Commun. 2016;7:11579.

16. Schiavon G, Hrebien S, Garcia-Murillas I, et al. Analysis of ESR1 mutation in circulating tumor DNA demonstrates evolution during therapy for metastatic breast cancer. Sci Transl Med. 2015;7(313):313ra182.

17. Takeshita T, Yamamoto Y, Yamamoto-Ibusuki M, et al. Analysis of ESR1 and PIK3CA mutations in plasma cell-free DNA from ER-positive breast cancer patients. Oncotarget. 2017;8(32):52142-52155.

18. Higgins JPT, Green S [webpage on the Internet]. Cochrane Handbook for Systematic Reviews of Interventions Version 5.1.0. The Cochrane Collaboration; 2011. Available from: http://handbook.cochrane.org/. Accessed May 17, 2015.

19. Parmar MK, Torri V, Stewart L. Extracting summary statistics to perform meta-analyses of the published literature for survival endpoints. Stat Med. 1998;17(24):2815-2834.

20. Tierney JF, Stewart LA, Ghersi D, Burdett S, Sydes MR. Practical methods for incorporating summary time-to-event data into meta-analysis. Trials. 2007;8:16.

21. Samphao S, Eremin JM, El-Sheemy M, Eremin O. Treatment of established breast cancer in post-menopausal women: role of aromatase inhibitors. Surgeon. 2009;7(1):42-55.

22. Francis PA, Regan MM, Fleming GF, et al; SOFT Investigators; International Breast Cancer Study Group. Adjuvant ovarian suppression in premenopausal breast cancer. $N$ Engl J Med. 2015;372(5): 436-446.

23. Yan S, Li K, Jiao X, Zou H. Tamoxifen with ovarian function suppression versus tamoxifen alone as an adjuvant treatment for premenopausal breast cancer: a meta-analysis of published randomized controlled trials. Onco Targets Ther. 2015;8:1433-1441.

24. Boér K. Fulvestrant in advanced breast cancer: evidence to date and place in therapy. Ther Adv Med Oncol. 2017;9(7):465-479.
OncoTargets and Therapy

\section{Publish your work in this journal}

OncoTargets and Therapy is an international, peer-reviewed, open access journal focusing on the pathological basis of all cancers, potential targets for therapy and treatment protocols employed to improve the management of cancer patients. The journal also focuses on the impact of management programs and new therapeutic agents and protocols on

\section{Dovepress}

patient perspectives such as quality of life, adherence and satisfaction. The manuscript management system is completely online and includes a very quick and fair peer-review system, which is all easy to use. Visit http://www.dovepress.com/testimonials.php to read real quotes from published authors. 